\title{
Correlatos Anatômico-Funcionais das Emoções Mapeados com TÉCNICAS de NeUROIMAGem FunCIONAL
}

\author{
Geraldo Busatto ${ }^{1}$, Jorge Cardoso de Almeida ${ }^{2}$, Carlos Toledo Cerqueira ${ }^{2}$ e \\ Clarice Gorenstein ${ }^{3}$ \\ Universidade de São Paulo - USP
}

\begin{abstract}
Nos dias de hoje, o uso de técnicas de neuroimagem funcional permite investigações detalhadas dos circuitos cerebrais engajados durante o desempenho de paradigmas de estimulação que envolvem diferentes aspectos do processamento emocional em seres humanos saudáveis. Este artigo discute os princípios básicos dos métodos mais comumente usados em estudos de neuroimagem funcional das emoções, faz uma revisão seletiva de achados recentes obtidos nestes estudos, fornece exemplos de novos paradigmas usados atualmente neste tipo de investigação, $e$ descreve modelos neuroanatômicos recentes para o processamento emocional formulados a partir dos achados da literatura contemporânea de neuroimagem. Por fim, enfatizamos o potencial das técnicas de neuroimagem funcional para ampliar o conhecimento a respeito da ação modulatória dos medicamentos usados para tratar condições mentais que alteram processos emocionais específicos, tais como a depressão e os transtornos de ansiedade.
\end{abstract}

Descritores: Imagem por Ressonância Magnética. Depressão (emoção). Ansiedade.

1 Docente do Departamento de Psiquiatria e Laboratório de Neuroimagem em Psiquiatria (LIM21) da Faculdade de Medicina - USP. Endereço eletrônico: geraldo.busatto@ honet.usp.br

2 Pós-Graduandos do Departamento de Psiquiatria e LIM21 da Faculdade de Medicina - USP.

3 Docente do Departamento de Farmacologia do Instituto de Ciências Biomédicas USP. Laboratório de Psicofarmacologia (LIM23) da Faculdade de Medicina - USP. 
Geraldo Busatto, Jorge C. Almeida, Carlos T. Cerqueira e Clarice Gorenstein

\section{Os primórdios das pesquisas de avaliação do funcionamento cerebral através de técnicas de neuroimagem}

partir da década de 1960, passou a ser possível usar, de forma válida e
lconfiável, métodos de neuroimagem funcional para avaliar padrões de funcionamento cerebral em seres humanos vivos e investigar anormalidades funcionais associadas a transtornos neuropsiquiátricos.

Até o final do século XX, os métodos de neuroimagem funcional mais comumente utilizados eram as técnicas de tomografia por emissão de pósitrons (positron emission tomography; PET) e a tomografia por emissão de fóton único (single photon computed tomography; SPECT). Ambas as técnicas, pertencentes ao campo da Medicina Nuclear, permitem a construção de mapas tridimensionais da atividade cerebral a partir da detecção de raios gama emitidos por traçadores marcados com isótopos radioativos, que são rapidamente captados pelo cérebro após administração venosa ou inalação. Dependendo do tipo de traçador marcado, pode-se obter imagens tomográficas do fluxo sangüíneo cerebral regional (FSCr), do metabolismo de glicose e da distribuição de receptores ou de terminais sinápticos cerebrais específicos.

Sabe-se, há mais de 100 anos, que mecanismos regulatórios no cérebro modificam o suprimento de sangue em resposta a variações locais no nível de atividade funcional (Roy \& Sherrington, 1890). Como o funcionamento cerebral depende basicamente do metabolismo oxidativo, e visto que nem oxigênio nem glicose podem ser aí armazenados, mudanças de FSCr em resposta a demandas funcionais são vistas como necessárias para suprir o tecido cerebral com estes substratos para o metabolismo energético (Raichle, 1987). Esta noção foi repetidamente confirmada nos estudos de PET cerebral em humanos, através da detecção de aumentos tanto de fluxo sangüíneo como de metabolismo de glicose durante tarefas de estimulação sensorial, motora ou cognitiva (Woods et al., 1991). Nota-se também um paralelismo grande entre os aumentos destas duas medidas, confirmando que ambas representam correlatos fiéis do funcionamento cerebral regional (Baron et al., 1980).

Pesquisas de FSCr e de metabolismo de glicose com PET e SPECT em amostras representativas de pacientes com transtornos neuropsiquiátricos 
contribuíram para um maior entendimento da fisiopatologia destes transtornos, identificando disfunções de áreas e circuitos cerebrais subjacentes aos sintomas mentais característicos dos mesmos. Nos primórdios do uso destas técnicas em Psiquiatria e Neurologia, o desenho experimental mais utilizado consistia na comparação dos padrões médios de funcionamento cerebral regional entre amostras de pacientes e grupos-controle de voluntários saudáveis estudados em repouso, sob estimulação sensorial ambiental mínima. Nesta linha, são clássicos achados como os de: diminuição de atividade em córtex frontal ("hipofrontalidade") em amostras de pacientes com esquizofrenia crônica (Andreasen et al., 1997); hipoatividade cortical em pacientes com Doença de Alzheimer, sobretudo em áreas têmporo-parietais e em relação direta com a intensidade dos déficits cognitivos da doença (Montaldi et al., 1990); alterações de atividade frontal em associação à intensidade do humor depressivo e retardo psicomotor em quadros depressivos maiores (Bench, Friston, Brown, Frackowiak, \& Dolan, 1993); e hiperatividade em córtex órbito-frontal, gânglios da base, cíngulo anterior e tálamo no transtorno obsessivo-compulsivo (Saxena, Brody, Schwartz, \& Baxter, 1998).

Com o decorrer do tempo, as técnicas de PET e SPECT passaram a ser utilizadas também para obter imagens durante a execução de paradigmas de ativação com estimulação sensorial, motora ou cognitiva, tipicamente contrastadas com imagens obtidas durante uma tarefa de base na mesma sessão experimental. Esta abordagem abriu um campo mais amplo para a investigação dos circuitos cerebrais implicados na execução de inúmeros processos mentais em seres humanos saudáveis. Além disso, estes estudos passaram a fornecer dados sobre os padrões de funcionamento cerebral associados a tarefas relevantes para a fisiopatologia de transtornos mentais específicos. São pioneiros nesta área os trabalhos de Weinberger, Berman e Zec (1986), que utilizando a técnica de FSCr após inalação de xenônio radioativo (precursora de PET e SPECT), identificaram atividade deficitária do córtex pré-frontal em pacientes portadores de esquizofrenia comparados com voluntários normais durante a execução do Wisconsin Card Sorting Test, tarefa neuropsicológica que demanda flexibilidade mental, engajando para isto regiões anteriores do córtex cerebral. Desde então, foram realizados inúmeros estudos de PET ou SPECT durante a execução de tarefas neuropsicológicas clássicas adaptadas para o contexto dos exames de neuroimagem, abrangendo memória, atenção, linguagem e outras operações 
mentais (Audenaert et al., 2002; Busatto et al., 1994; Cabeza \& Nyberg, 2000; Elliot, Sahakian, Michael, Paykel, \& Dolan, 1998).

\section{O desenvolvimento da Ressonância Magnética Funcional}

O campo dos estudos de neuroimagem funcional durante paradigmas de estimulação foi revolucionado a partir da década de 1990, com o desenvolvimento de técnicas que permitem a avaliação da atividade funcional do cérebro utilizando os princípios básicos de aquisição de imagens de ressonância magnética. O primeiro desenvolvimento nesta área veio com o uso de injeções em bolo de contrastes paramagnéticos como gadolínio para o imageamento da vasculatura cerebral (Belliveau et al., 1991). Mais recentemente, vêm sendo usadas técnicas muito mais sofisticadas e completamente não invasivas, utilizando imagens de contraste dependente dos níveis de oxigenação do sangue ("blood-oxygenation level dependent effect" ou efeito BOLD) (Amaro Jr. \& Yamashita, 2001).

O efeito BOLD é oriundo da observação de que alterações na proporção relativa no sangue entre hemoglobina oxigenada (que contém ferro diamagnético) e hemoglobina de-oxigenada (que contém ferro paramagnético) podem ser detectadas como variações de sinal em imagens de ressonância magnética (Ogawa, Lee, Kay, \& Tank, 1990). Sabe-se, como já mencionado anteriormente, que aumentos de fluxo e volume sangüíneo ocorrem em resposta a aumentos na demanda funcional local (por exemplo, durante uma tarefa mental). Este aumento de fluxo sangüíneo traz consigo um aumento considerável no aporte de oxigênio, mas por outro lado, o aumento na extração de oxigênio pelo tecido cerebral é bastante discreto (Fox \& Raichle, 1986). O excesso de oxigênio sangüíneo leva a uma diminuição relativa da concentração de deoxi-hemoglobina em capilares e vênulas que drenam o sangue do tecido, e esta mudança é detectada como aumento do sinal nas imagens. Obtém-se assim uma mudança de sinal que reflete de forma confiável as variações da demanda funcional local do tecido cerebral (Ogawa et al., 1990).

Mudanças no sinal de ressonância magnética provenientes do efeito BOLD são transitórias e sutis. Com o desenvolvimento de bobinas para imageamento "eco-planar" ("echo-planar imaging") capazes de adquirir imagens ultra- 
rápidas do cérebro inteiro, é possível a obtenção de seqüências de cortes seriados em intervalos da ordem de poucos segundos (Amaro Jr. \& Yamashita, 2001). A partir de então, tal hardware passou a integrar a maioria de equipamentos de ressonância magnética, e com isto vem sendo possível a realização de um grande número de pesquisas, em voluntários normais e pacientes com transtornos neuropsiquiátricos, realizados durante uma gama variada de tarefas de estimulação sensoriomotora, cognitiva e emocional (Beauregard et al., 1998; Breiter et al., 1996b; Broome et al., 2004; Busatto, et al., 1997; Cabeza \& Nyberg, 2000; Moll et al., 2002; Nolde, Johnson, \& D'Esposito, 1998). Estes estudos têm não só replicado achados anteriores da literatura de PET e SPECT, mas também descrito novos achados não acessíveis com as técnicas convencionais, gerando novas hipóteses sobre o funcionamento mental humano durante tarefas específicas, e sobre a fisiopatologia dos transtornos de base cerebral.

As vantagens da Ressonância Magnética Funcional (RMf) sobre os métodos convencionais de PET e SPECT são várias, a começar por se tratar de uma técnica totalmente não invasiva, que permite um número amplo de experimentos por sujeito, dentro da mesma sessão ou em períodos diferentes ao logo de horas, dias ou meses. Além disso, a resolução temporal da técnica de RMf é muito superior às técnicas de Medicina Nuclear e suas imagens podem também ter considerável resolução espacial, trazendo ainda a possibilidade de co-registro de RM estrutural de altíssima resolução, sem mudar o posicionamento do sujeito.

É viável realizar um experimento completo de RMf durante estimulação mental em poucos minutos, durante os quais pode-se adquirir cem ou mais sequiências de múltiplos cortes cerebrais. No desenho mais convencional, os paradigmas de estimulação são organizados em "blocos" de duas tarefas diferentes que se alternam ao longo do tempo, uma das quais envolvendo as operações mentais de interesse e uma outra que funciona como tarefa-controle. Diversas execuções das mesmas tarefas ocorrem ao longo do experimento, e são usados métodos estatísticos na fase do processamento dos dados, para detectar os locais cerebrais nos quais houve mudanças significativas de sinal BOLD em consonância com a realização de cada uma das tarefas (Senaha et al., 2005).

Um tipo de estratégia de RMf alternativa ao desenho convencional "em bloco" é a chamada RMf evento-relacionada ("event-related fMRI”) (Buckner, 
1998; D’Esposito, Zarahn, \& Aguirre, 1999). Em geral, como a técnica de RMf permite a aquisição de cortes individuais do encéfalo em frações de segundo, é possível detectar, de forma confiável, mudanças de sinal BOLD dentro desta janela mínima de tempo. Partindo desta premissa, a RMf evento-relacionada utiliza desenhos experimentais nos quais as imagens são adquiridas e analisadas de forma a se detectar padrões de atividade funcional durante diferentes etapas de uma mesma operação mental (por exemplo, durante diferentes etapas do processamento de memória, incluindo a apresentação de novas informações, estocagem, e rememoração dos dados) (Wheeler et al., 2006).

\section{Métodos para análise de imagens de ressonância magnética funcional, PET e SPECT}

Na rotina clínica diária de Radiologia ou Medicina Nuclear, a avaliação de imagens cerebrais é baseada na inspeção visual das mesmas. Já em estudos de pesquisa na área neuropsiquiátrica, como discutido acima, os desenhos experimentais envolvem mais comumente comparações estatísticas de médias entre um grupo de pacientes com um determinado transtorno versus um grupo de voluntários normais, ou então, avaliações estatísticas das mudanças de padrões nas imagens dos mesmos sujeitos estudados em diferentes condições ao longo do tempo.

Em décadas recentes, técnicas sofisticadas de processamento de imagens e análise estatística dos dados vêm sendo desenvolvidas, comumente denominadas métodos de análise voxel a voxel. Neste tipo de técnica, as imagens passam por algumas transformações antes das comparações estatísticas entre grupos, entre as quais estão incluídas: etapas de rotação e alinhamento entre as imagens dos diferentes sujeitos de cada grupo; e deformações discretas das imagens para conformá-las a um modelo anatômico padronizado. Após tais tipos de processamento, pode-se pressupor que cada unidade tridimensional do volume total adquirido em um indivíduo (o voxel, que é em geral cúbico e com poucos $\mathrm{mm}^{3}$ de volume) tem a mesma localização em todos os sujeitos do estudo, localização esta definida por coordenadas milimétricas nos eixos x, y, e z do espaço tridimensional padronizado. Por fim, através da computação das comparações estatísticas entre grupos para cada voxel, pode-se produzir 
Correlatos Anatômico-Funcionais das Emoções Mapeados com Técnicas de...

mapas tridimensionais que mostram a significância estatística, a localização cerebral e a extensão dos grupamentos de voxels que apresentaram diferença significativa entre os grupos no limiar estatístico escolhido.

\section{O uso da técnica de PET na indução de emoções}

Durante a maior parte do século XX, estudos em pacientes com lesões cerebrais e em animais estabeleceram as bases da investigação da neuroanatomia da regulação das emoções.

A partir da década de 1990, passou-se a acreditar que este campo do conhecimento poderia ser objeto de interesse também da neuroimagem, quando foram realizados os primeiros estudos de PET em amostras de pacientes com transtornos neuropsiquiátricos usando como paradigma de estimulação a própria provocação de emoções e sintomas afetivos. Tais estudos documentaram, pela primeira vez, os padrões de atividade cerebral associados, por exemplo, à eliciação de sintomas obsessivo-compulsivos, a picos de ansiedade em pacientes fóbicos ou com transtorno do estresse pós-traumático (Rauch et al., 1997), e reações emocionais em pacientes com transtornos do humor (George, Ketter, \& Post, 1994).

Após os estudos iniciais acima, abriu-se uma ampla gama de investigações de neuroimagem destinadas a mapear os circuitos cerebrais envolvidos no processamento emocional humano, predominantemente em amostras de indivíduos saudáveis. Para isso, vários paradigmas foram desenvolvidos com o objetivo de evocar diferentes respostas emocionais, procurando correlacionar os circuitos cerebrais ativados com as emoções produzidas. Alguns tipos de paradigmas de estimulação utilizados e os resultados obtidos estão exemplificados abaixo.

Processamento de emoções relacionadas a diferentes expressões faciais. Uma das estratégias de mais utilidade para estudar o processamento de emoções durante experimentos de neuroimagem funcional tem sido a de apresentar faces que expressam, em diferentes graus, emoções específicas como medo, raiva, nojo, etc. Por exemplo, num dos primeiros estudos de PET nesta linha, Morris et al. (1996) observaram que expressões faciais de medo provo- 
caram maior aumento de fluxo na amídala esquerda comparado à observação de faces que expressavam alegria. No entanto, deve-se fazer a ressalva que os estudos que apresentam diferentes fotos com expressões faciais não evocam necessariamente emoções, podendo, alternativamente, representar simplesmente a percepção de equivalentes emocionais.

Estudos durante rememoração de eventos tristes. Pardo, Pardo e Raichle (1993) detectaram aumentos transitórios de fluxo sanguiíneo no córtex órbito-frontal esquerdo e no córtex pré-frontal quando voluntários normais eram induzidos a imaginar ou recordar uma situação na qual poderiam se sentir muito tristes ("melancolia transitória"). Mayberg et al. (1999) provocaram sentimentos de tristeza em voluntários normais através da evocação de experiências pessoais e contrastaram os resultados com os de um outro experimento anterior do mesmo grupo, no qual pacientes em quadros depressivos maiores haviam sido avaliados antes e após a remissão dos sintomas. Na condição de tristeza nos voluntários saudáveis, os autores observaram aumento de $\mathrm{FSCr}$ na ínsula anterior e no córtex cingulado subgenual, além de diminuições de FSCr no córtex pré-frontal dorsolateral e parietal inferior à direita. Os autores propuseram a existência de um padrão de reciprocidade funcional entre áreas límbicas e pré-frontais mediando tanto as mudanças de humor transitórias em indivíduos sadios, como as crônicas, patológicas que caracterizam episódios depressivos maiores (Mayberg et al., 1999).

Estudos de rememoração de eventos felizes. George et al. (1995) relataram uma diminuição do FSCr na região superior do córtex pré-frontal direito com a indução transitória de sensação de alegria e bem-estar, através da rememoração de eventos pessoais e visualização/reconhecimento de faces com expressão de felicidade. Num estudo que usou métodos similares numa amostra maior de indivíduos saudáveis, Damásio et al. (2000) reportaram mudanças na atividade funcional dos gânglios da base, do hipocampo e do córtex pré-frontal durante a lembrança de episódios felizes da vida pregressa dos indivíduos. Em um outro estudo de PET realizado por Lane et al. (1997), o uso de filmes e lembrança de eventos passados de conteúdo feliz levou a aumentos da atividade do tálamo, do córtex pré-frontal medial e de porções do córtex temporal. 
Correlatos Anatômico-Funcionais das Emoções Mapeados com Técnicas de...

\section{O uso da técnica de RMf na indução de emoções}

Nos últimos anos, o desenvolvimento e o uso rotineiro da RMf em centros de pesquisa internacionais levou literalmente a uma explosão de artigos científicos reportando resultados de estudos utilizando esta técnica na avaliação de emoções. Seguem abaixo alguns exemplos destes estudos, seguindo os mesmos tópicos selecionados para a nossa breve revisão de estudos de FSCr por PET feita no item anterior.

Processamento da emoção relacionada a diferentes expressões faciais. Num dos primeiros estudos de provocação de emoções usando RMf, Breiter et al. (1996a) replicaram o achado com PET de Morris et al. (1996) de que expressões faciais de medo provocam maior aumento de fluxo na amídala esquerda e mostram uma rápida habituação da resposta (ausência de ativação com a repetição do estímulo). Já Phillips et al. (1997) mostraram que a observação de expressão de nojo ("disgust”) não ativou a amídala, mas sim a ínsula anterior, região implicada no processamento de estímulos gustativos. Num estudo subseqüiente que tirou vantagem da excelente resolução temporal da técnica de RMf, Whalen et al. (1998) mostraram que é possível ativar a amídala durante a apresentação de faces expressando emoções mesmo sem os indivíduos tomarem consciência da apresentação do estímulo visual; para tanto, os autores apresentaram tais faces durante períodos curtíssimos de tempo (33 mili-segundos), insuficientes para que a estimulação de medo fosse conscientemente percebida pelos sujeitos. É interessante notar que neste estudo a ativação da amídala foi maior durante a apresentação de faces de medo em comparação com faces felizes, dando novamente suporte à noção de que o nível de ativação desta estrutura límbica depende da valência emocional do estímulo apresentado (Whalen et al., 1998). Deste ponto em diante, um amplo número de estudos de RMf vem sendo realizados em indivíduos saudáveis, confirmando a noção de que a atividade funcional de diferentes áreas límbicas e/ou neocorticais é modulada de acordo com o significado emocional das faces apresentadas (Vuilleumier \& Pourtois, 2007). Além disso, este tipo de paradigma tem também sido utilizado com sucesso para demonstrar alterações na atividade funcional de regiões cerebrais envolvidas no processamento emocional de faces em portadores de transtornos mentais; um exemplo interessante é fornecido pelo estudo de Stein et al. (2002), no qual observou-se 
hiperatividade da amídala durante a apresentação de expressões faciais pouco amistosas a um grupo de sujeitos portadores de fobia social comparados a controles assintomáticos.

Estudos durante indução de tristeza. Num estudo pioneiro de RMf, Beauregard et al. (1998) induziram emoções de tristeza em voluntários normais e pacientes depressivos unipolares durante a apresentação de um filme de forte conteúdo emocional. Ambos os grupos apresentaram ativação do córtex pré-frontal medial e inferior, córtex temporal médio, núcleo caudado e cerebelo. No entanto, houve ativação maior em córtex pré-frontal medial esquerdo e cíngulo anterior direito em pacientes deprimidos, sugerindo um papel crítico para estas duas regiões na fisiopatologia da depressão maior. Em anos subsequientes, diversos outros estudos usando paradigmas semelhantes vêm sendo reportados em amostras de indivíduos saudáveis (Phan et al., 2002) e pacientes com depressão maior (Beauregard, Paquette, \& Levesque, 2006).

Estudos durante indução de felicidade. Esta tem sido uma área ainda escassamente investigada com a RMf. Num dos poucos estudos realizados, Markowitsch, Vandekerckhove, Lanfermann e Russ (2003) reportaram aumentos de atividade funcional no cíngulo anterior, amídala dorsal, pálido ventral, precúneo e áreas de neocórtex frontal e temporal durante indução de felicidade despertada por palavras apresentadas visualmente.

\section{Estudos de PET e RMf durante provocação de emoçães: síntese e formulação de modelos cerebrais do processamento emocional}

Como dito anteriormente, a literatura na área de estudos de neuroimagem funcional durante paradigmas de estimulação emocional cresceu de forma muito significativa nos últimos anos, tanto no que diz respeito a estudos em voluntários saudáveis quanto àqueles realizados em amostras de sujeitos portadores de transtornos neuropsiquiátricos. Não só os estados emocionais de tristeza e felicidade mencionados nos itens anteriores, mas também vários outros como medo, raiva, ansiedade e nojo têm sido avaliados, induzidos por paradigmas envolvendo o uso de estímulos impessoais (como apresentações de faces ou fotos e filmes), ou através de paradigmas que utilizam estímulos 
Correlatos Anatômico-Funcionais das Emoções Mapeados com Técnicas de...

pessoais (tais como os relatos autobiográficos de conteúdo emocional descritos em itens anteriores).

Em síntese, independentemente do tipo de paradigma utilizado em voluntários normais, os estudos de neuroimagem funcional salientam as seguintes regiões cerebrais como as principais áreas envolvidas ma regulação do humor e geração de emoções (negativas e positivas): córtex pré-frontal dorsolateral e medial; córtex órbito-frontal; amídala; cíngulo anterior; corpo estriado; e córtex insular. Além disso, os dados de neuroimagem sugerem que há relações mais específicas entre algumas regiões cerebrais e determinados tipos de emoções, tais como o envolvimento da amídala no processamento de emoções de medo, e o envolvimento da porção subcalosa do giro do cíngulo no processamento de emoções de tristeza. Por fim, as pesquisas de neuroimagem têm confirmado a noção de que há um grau considerável de coincidência entre os circuitos cerebrais engajados durante o processamento emocional normal e o processamento emocional alterado em pacientes com transtornos psiquiátricos, tais como os depressivos e os ansiosos (Phillips, Drevets, Rauch, \& Lane, 2003b).

O grande volume da literatura acima impede uma revisão detalhada dos resultados obtidos num artigo como este. Tal tipo de revisão cabe mais a estudos meta-analíticos de achados de neuroimagem funcional durante processamento emocional, que já vêm sendo publicados em periódicos de destaque (Phan, Wager, Taylor, \& Liberzon, 2002). Estas meta-análises dão indicações claras de quão grande tem sido a contribuição dos estudos de neuroimagem funcional para a formulação contemporânea de modelos cerebrais para o processamento emocional.

Recentemente, o processamento emocional tem sido separado em diferentes elementos, incluindo: (a) uma etapa de identificação do significado emocional de um determinado estímulo; (b) a emergência propriamente dita do estado afetivo e das modificações neurovegetativas associadas, em consonância com o significado do estímulo; e (c) a modulação da resposta afetiva, de forma que a resposta comportamental do indivíduo seja contextualmente apropriada (Phillips, Drevets, Rauch, \& Lane, 2003a).

Com base principalmente em achados recentes de neuroimagem, leva-se hoje em dia em conta a existência de dois sistemas neurais (ventral e 
dorsal), que seriam responsáveis pelas diferentes etapas da geração e controle de estados afetivos. O sistema ventral, composto por circuitos envolvendo amídala, ínsula, corpo estriado ventral, regiões ventrais do cíngulo anterior e córtex órbito-frontal, estaria relacionado às etapas de identificação do significado emocional de estímulos (estresse), e de produção dos estados afetivos específicos em resposta a tais estímulos. Já o sistema dorsal, composto pelo hipocampo, por regiões dorsais do cíngulo anterior e pelo córtex pré-frontal, estaria relacionado à regulação dos estados afetivos, envolvendo a necessária modulação dos processos acima para a obtenção das respostas comportamentais contextualmente apropriadas. $\mathrm{O}$ funcionamento equilibrado desses dois sistemas depende de conexões recíprocas existentes entre eles, o que permite a modulação mútua para gerar comportamentos e emoções complexos. Enquanto o sistema ventral recebe aferências extensas de áreas sensoriais primárias e de associação, o dorsal está mais relacionado a mecanismos cognitivos, como memória e atenção (Phillips et al., 2003a).

\section{Um exemplo prático de aplicação da RMf em nosso meio - paradigma de indução de irritabilidade através da apresentação de textos pessoais}

Nosso grupo teve a oportunidade participar do "Estudo Psicobiológico da Regulação Emocional a partir dos Efeitos de Antidepressivos", realizado no Instituto de Psiquiatria do HCFMUSP com o apoio da FAPESP (projeto temático 01/00189-9), que tem como objetivo principal contribuir para a compreensão dos mecanismos de regulação do humor normal. Dentro de um módulo de neuroimagem deste projeto, formulamos dois paradigmas de estimulação emocional a serem usados durante aquisição de dados de RMf - indução de irritabilidade por meio de textos pessoais (descrito abaixo) e provocação de emoções através de estímulos fotográficos (descrito mais adiante). Usando tais paradigmas, as imagens de RMf são adquiridas em voluntários normais sob condições basais e tratados com doses baixas de um antidepressivo, a clomipramina.

Um dos possíveis métodos para indução de irritabilidade, que se assemelha aos métodos "clássicos" de indução de resposta emocional de outros 
Correlatos Anatômico-Funcionais das Emoções Mapeados com Técnicas de...

tipos, incluindo tristeza, ansiedade e raiva (Mayberg et al., 1999; Pardo et al., 1993), é a apresentação de textos descrevendo situações e eventos que tipicamente levam a irritabilidade naquele sujeito. Neste caso, os textos apresentados são verdadeiramente pessoais contendo os elementos comuns aos eventos que geram irritação nos sujeitos selecionados.

No nosso estudo, são inicialmente identificadas para cada sujeito situações de sua vida que eliciam estados de maior irritabilidade (por exemplo, barulho enquanto realizando uma tarefa que exige concentração, perda de tempo em trânsito quando muito ocupado). Exemplos de situações cotidianas podem ser extraídas do instrumento "Hassles and Uplifts scale" (DeLongis, Folkman, \& Lazarus, 1988). A seleção da situação alvo para cada sujeito é feita a partir das informações obtidas através de entrevista semi-estruturada preparada com o objetivo de localizar situações-alvo de irritabilidade, neutras e positivas para cada indivíduo (priorizando aquelas que ocorreram preferencialmente nos 6 meses anteriores). É importante também avaliar a capacidade do sujeito para se imaginar visualmente nas situações a serem descritas nos scripts, o que pode ser feito com o auxílio de um questionário ("Vividness of Visual Imagery Questionnaire"; Marks, 1989). A entrevista é gravada, e após a seleção do tipo de situação alvo para um dado sujeito, o mesmo é encorajado a descrever uma ocorrência real recente neste tipo de situação em sua vida. É necessário identificar uma situação rica em detalhes, e de duração suficientemente longa para permitir a adaptação da mesma ao contexto de aquisição das imagens de RMf. A partir do material obtido com o sujeito, é construído um texto narrando em detalhe a experiência escolhida. $\mathrm{O}$ texto preparado deve ser gravado em estúdio especializado por um locutor profissional gênero específico (mulher para sujeitos do sexo feminino) e armazenado para posterior apresentação em Compact Disc.

Além dos scripts de irritabilidade, são apresentados, dentro da mesma sessão experimental, scripts autobiográficos de situações de felicidade (valência positiva) e situações corriqueiras/habituais (valência neutra), a fim de permitir que sejam contrastados os padrões de ativação cerebral correspondentes aos diferentes tipos de resposta emocional e componentes da tarefa de estimulação. As situações que compõem a narrativa de experiência pessoal neutra, com potencial baixo de induzir irritabilidade ou outras emoções, e de felicida- 
de são selecionadas na mesma entrevista inicial. Os textos construídos a partir destas narrativas são gravados da mesma maneira que o texto de indução de irritabilidade, e devem ter a mesma duração.

Por fim, são usadas também vinhetas curtas, neutras e não-pessoais, antes da apresentação de cada tipo de script pessoal, como tarefa-controle adicional e com o objetivo de contribuir para a dissipação da emoção despertada pelo texto autobiográfico apresentado imediatamente antes. Os scripts neutros não pessoais são formulados a partir de materiais da imprensa comum, livros, jornais e revistas, obedecendo aos critérios idênticos ao dos scripts neutros pessoais. Estes scripts são idênticos para todos os sujeitos no seu conteúdo e na forma de apresentação.

Durante a aquisição das imagens de RMf, os sujeitos permanecem com os olhos fechados, e escutam através de fones de ouvido desmagnetizados próprios para uso no ambiente do equipamento de ressonância magnética os textos preparados acima. É solicitado que os sujeitos "entrem" na situação imaginando que aquilo está realmente acontecendo com eles, e não reprimam qualquer sentimento ou emoção que emergir.

A seqüência de cada bloco é composta por um script positivo, um neutro e um de irritabilidade, apresentados sempre nesta ordem, cada um precedido por 20 segundos de texto neutro não-pessoal. São realizados 3 blocos dentro de uma mesma sessão experimental, com intervalo de 2 minutos entre si. Tal repetição é fundamental para permitir que sejam calculados valores médios dos padrões de ativação cerebral regional durante cada tipo de resposta emocional, e com isto aumentar o poder estatístico durante a análise dos dados de imagem.

Ao final dos scripts, são apresentadas escalas discretas de avaliação, através das quais os indivíduos indicam, de 1 a 4, o grau de intensificação de emoções após a apresentação de cada texto. Para tal fim, é usada uma "luva" através da qual os sujeitos respondem às escalas analógicas visuais escolhendo um entre 4 dedos preparados para registrar respectivamente os escores 1, 2, 3 ou 4 para cada escala, de forma rápida e demandando pouco esforço. Além disso, a intensidade dessa resposta emocional subjetiva pode ser confrontada com a resposta autonômica a ela concomitante (p.ex., registro simultâneo de freqüência cardíaca, resposta galvânica da pele). 
Correlatos Anatômico-Funcionais das Emoções Mapeados com Técnicas de...

\section{Provocação de emoções por meio de estímulos fotográficos - mais um exemplo prático}

Um dos métodos amplamente explorados na literatura até o momento em estudos de neuroimagem funcional para a provocação de emoções é o de apresentação de fotografias de conteúdo emocional significativo, sendo o banco de fotografias mais utilizado o International Affective Picture System (IAPS, Lang, Bradley, \& Cuthbert, 2001).

O paradigma que construímos consiste da apresentação de seqüência de fotografias do IAPS que sejam capazes de despertar emoções como raiva, medo ou felicidade, em sequiências separadas. Dentro deste paradigma, as fotos de cada uma das categorias emocionais são contrastadas com fotos neutras do IAPS que são pareadas para complexidade visual entre si e com cada um dos 3 grupos de imagens de conteúdo emocional. O uso de fotos neutras como controle universal das outras tarefas permite que sejam contrastados os padrões de ativação cerebral correspondentes a cada tipo de resposta emocional versus o padrão de ativação pela estimulação visual simples com as imagens neutras.

Para a sua realização durante a aquisição de imagens de RMf, o experimento é idealizado em 3 etapas, cada qual contrastando um tipo específico de fotografia de conteúdo emocional (p.ex., raiva, medo ou felicidade) versus imagens neutras do IAPS. Dentro de cada etapa, são apresentados 5 blocos de fotografias do tipo de emoção selecionada, alternados com 3 blocos de fotos neutras. Cada bloco contém 3 fotos de um determinado tipo de emoção, apresentadas durante 8 segundos cada (24 segundos por bloco). Assim, para cada tipo de emoção, há um total de 8 blocos, incluindo 15 fotos de conteúdo emocional e 9 fotos neutras.

Com o objetivo de maximizar a chance de se despertar a emoção desejada durante a apresentação das fotos, usamos a estratégia originalmente empregada por Phillips et al. (2000) em estudos recentes de RMf em pacientes com transtorno obsessivo-compulsivo. Dentro desta proposta, os indivíduos são instruídos a se imaginar vivendo o tipo de situação que será mostrada nas fotos visualizadas em seguida. Por exemplo, na apresentação de fotos de animais selvagens que objetivam despertar medo, o sujeito recebe antes uma ins- 
trução do tipo: "Imagine estes animais diante de você". Estas instruções podem ser fornecidas visualmente na mesma tela de computador que mostra as fotos, durante 6 segundos antes da apresentação de cada sequiência de 3 imagens. As instruções são mudadas para cada bloco de 3 fotos de um mesmo tipo de emoção, para serem especificamente compatíveis com o tema predominante nas fotos agrupadas naquele bloco. Para registrar a natureza e a intensidade da resposta emocional predominante durante a apresentação das fotos, apresentase, após cada bloco de 3 fotografias, escalas analógicas visuais avaliando respectivamente as emoções de medo, raiva, felicidade e ativação ("arousal"). As respostas a estas escalas são fornecidas pelo sujeito utilizando a mesma "luva" mencionada no item anterior.

\section{Avaliação de mudanças no funcionamento cerebral após tratamento medicamentoso}

Os paradigmas citados acima estão sendo atualmente empregados pelo nosso grupo com a finalidade de avaliar mudanças na atividade funcional do cérebro humano após o uso de medicamentos de ação no sistema nervoso central.

A área de investigação de mudanças na atividade de circuitos cerebrais envolvidos no processamento emocional após o uso de medicamentos vem sendo considerada um dos campos de maior alcance atual dos estudos de neuroimagem funcional durante processamento emocional. Um exemplo clássico desta linha são os estudos de PET e SPECT realizados em repouso, com traçadores para FSCr ou metabolismo de glicose, em sujeitos com sintomas depressivos maiores estudados antes e após tratamento com antidepressivos (Mayberg et al., 1999). Tais estudos mostram que sinais de hipoatividade em áreas do córtex frontal associados aos sintomas depressivos se revertem após o tratamento eficaz. Em outros estudos, têm sido verificado também o envolvimento de áreas cerebrais adicionais especificamente em associação com a resposta ao tratamento medicamentoso antidepressivo, como a porção rostral do cíngulo anterior (Mayberg et al., 2000). Há, inclusive, especulações de que padrões de atividade pré-tratamento nesta última região cerebral poderão ser usados no futuro para prever se um indivíduo teria uma resposta favorável ou não a tratamentos medicamentosos para sintomas depressivos. 
Em estudos recentes de RMf, vêm sendo demonstradas mudanças de atividade funcional em pacientes com sintomas depressivos após tratamento medicamentoso durante a execução de paradigmas sofisticados de estimulação emocional, tais como reconhecimento de faces expressando tristeza $(\mathrm{Fu}$ et al., 2004) e visualização de fotografias do IAPS com conteúdo emocional (Schaefer, Putnam, Benca, \& Davidson, 2006). Tais estudos confirmam e ampliam os achados anteriores de mudanças de atividade funcional em regiões neocorticais, límbicas e paralímbicas do cérebro, e têm grande potencial para ajudar a desvendar os mecanismos cerebrais através dos quais os tratamentos medicamentosos normalizam processos emocionais e cognitivos anormais que acometem pacientes com quadros depressivos e ansiosos.

Deve-se salientar, no entanto, uma importante limitação destas pesquisas. Por envolverem amostras de sujeitos clinicamente deprimidos, tais estudos não podem distinguir se as mudanças de atividade cerebral pré- versus pós-tratamento capturadas nas imagens representam efeitos diretos e específicos dos medicamentos sobre as regiões relevantes para o processamento emocional, ou simplesmente refletem a mudança no estado sintomatológico dos pacientes. A fim de eliminar o viés causado por tal melhora sintomatológica, estudos recentes de RMf têm investigado padrões de mudança na atividade cerebral decorrente do uso de medicamentos em voluntários assintomáticos. Num estudo recente deste tipo, Harmer e colaboradores avaliaram voluntários saudáveis sob uso de citalopram, medicamento inibidor seletivo de recaptura da serotonina, ou de placebo, durante paradigma de processamento de faces (Harmer, Mackay, Reid, Cowen, \& Goodwin, 2006). Os autores registraram diferenças na atividade funcional entre sujeitos sob uso de citalopram versus placebo em duas regiões relevantes para o processamento emocional, a amídala e o córtex prefrontal medial. Estes resultados confirmam a noção de que o uso do medicamento modula, de forma específica, a atividade neuronal de regiões de importância crítica para a regulação emocional.

Busatto, G., Almeida, J. C., Cerqueira, C. T., \& Gorenstein, C. (2006). Neuroanatomical correlates of emotions mapped with functional neuroimaging techniques. Psicologia USP, 17(4), 135-157. 


\begin{abstract}
Nowadays, the use of functional imaging techniques allows detailed investigations of the brain circuits engaged during the performance of stimulation paradigms that involve different aspects of emotional processing in the healthy human brain. This article discusses the basic principles of the methods most often employed in functional neuroimaging studies of emotion, selectively reviews recent findings obtained in these studies, provide examples of new paradigms currently being used in such kind of investigation, and outlines recent neuroanatomical models of emotional processing that have emerged from the contemporary functional imaging literature. Finally, we emphasize the potential of functional imaging techniques to increase knowledge about the modulatory action of medications used to treat mental conditions that alter specific emotional processes, such as depression and anxiety disorders.
\end{abstract}

\title{
Index terms: Magnetic Resonance Imaging. Depression (emotion). Anxiety.
}

Busatto, G., Almeida, J. C., Cerqueira, C. T., \& Gorenstein, C. (2006). Les corrélations anatomique-foncionelles des émotions tracées avec des techniques de sésonance magnétique fonctionnelle. Psicologia USP, 17(4), 135-157.

Résumé: De nos jours, l'utilisation des techniques de neuroimage fonctionnelle permet des investigations détaillées sur les circuits de cerveau engagés pendant l'exécution des paradigmes de stimulation qui impliquent différents aspects du traitement émotif dans les etres humains sains. Cet article presente les principes de base des méthodes le plus souvent utilisées dans des études de neuroimage fonctionnelle des émotions, revise sélectivement les résultats récents obtenus dans les études de cette nature, fournis des exemples de nouveaux paradigmes actuellement employés dans un tel genre de recherche, et decrive les modèles neuroanatomiques récents pour le traitement émotif formulés ayant pour base la littérature contemporaine de neuroimage. En conclusion, nous soulignons le potentiel des techniques de neuroimage fonctionnelle pour faire monter la connaissance au sujet de l'action modulatoire des médicaments employés pour traiter les conditions mentales qui changent les processus émotifs spécifiques, tels la dépression et l'anxieté.

Mots-clés: Résonance Magnétique. Dépression. Anxiété. 
Correlatos Anatômico-Funcionais das Emoções Mapeados com Técnicas de...

\section{Referências}

Amaro Jr., E., \& Yamashita, H. (2001). Aspectos básicos de tomografia computadorizada e ressonância magnética. Revista Brasileira de Psiquiatria, 23(Supl. 1), 2-3.

Andreasen, N. C., O’Leary, D. S., Flaum, M., Nopoulos, P., Watkins, G. L., Boles Ponto, L. L. et al. (1997). Hypofrontality in schizophrenia: Distributed dysfunctional circuits in neuroleptic-naive patients. Lancet, 349, 1730-1734.

Audenaert, K., Goethals, I., Van Laere, K., Lahorte, P., Brans, B., Versijpt, J. et al. (2002). SPECT neuropsychological activation procedure with the Verbal Fluency Test in attempted suicide patients. Nuclear Medicine Communications, 23, 907-916.

Baron, J. C., Rougemont, D., Collard, P., Bustany, P., Bousser, M. G., \& Comar, D. (1980). Coupling between cerebral blood flow, oxygen consumption, and glucose utilization: Its study with positron emission tomography. In M. Reivich \& A. Alavi (Eds.), Positron emission tomography (pp. 203-218). New York: Alan R. Liss.

Beauregard, M., Leroux, J. M., Bergman, S., Arzoumanian, Y., Beaudoin, G., Bourgouin, P. et al. (1998). The functional neuroanatomy of major depression: An fMRI study using an emotional activation paradigm. Neuroreport, 9, 3253-3258.

Beauregard, M., Paquette, V., \& Levesque, J. (2006). Dysfunction in the neural circuitry of emotional self-regulation in major depressive disorder. Neuroreport, 17, 843-846.

Belliveau, J. W., Kennedy, D. N. Jr., McKinstry, R. C., Buchbinder, B. R., Weisskoff, R. M., Cohen, M. S. et al. (1991). Functional mapping of the human visual cortex by magnetic resonance imaging. Science, 254(5032), 716-719.

Bench, C. J., Friston, K. J., Brown, R. G., Frackowiak, R. S., \& Dolan, R. J. (1993). Regional cerebral blood flow in depression measured by positron emission tomography: The relationship with clinical dimensions. Psychological Medicine, 23, 579-590.

Breiter, H. C., Etcoff, N. L., Whalen, P. J., Kennedy, W. A., Rauch, S. L., Buckner, R. L. et al. (1996a). Response and habituation of the human amygdala during visual processing of facial expression. Neuron, 17, 875-887.

Breiter, H. C., Rauch, S. L., Kwong, K. K., Baker, J. R., Weisskoff, R. M., Kennedy, D. N. et al. (1996b). Functional magnetic resonance imaging of symptom provocation in obsessive compulsive disorder. Archives of General Psychiatry, 53, 595-606.

Broome, M. R., Matthiasson, P., Chitnis, X., Picchioni, M., Woolley, J. B., \& Brett, C. (2004). Functional imaging in early psychosis: An fMRI study of prodromal and first-episode subjects on motor, verbal and memory tasks. Schizophrenia Research, 70(Suppl. 1), 112.

Buckner, R. L. (1998). Event-related fMRI and the hemodynamic response. Human Brain Mapping, 6, 373-377. 
Busatto, G. F., Costa, D. C., Ell, P. J., Pilowsky, L. S., David, A. S., \& Kerwin, R. W. (1994). Regional cerebral blood flow $(\mathrm{rCBF})$ in schizophrenia during verbal memory activation: A 99mTc-HMPAO single photon emission tomography (SPET) study. Psychological Medicine, 24, 463-472.

Busatto, G., Howard, R. J., Há, Y., Brammer, M., Wright, I., Woodruff, P. W. et al. (1997). A functional magnetic resonance imaging study of episodic memory. Neuroreport, 8 , 2671-2675.

Cabeza, R., \& Nyberg, L. (2000). Imaging cognition II: An empirical review of 250 PET and fMRI studies. Journal of Cognitive Neuroscience, 12, 1-47.

Damasio, A. R., Grabowski, T. J., Bechara, A., Damasio, H., Ponto, L. L., Parvizi, J. et al. (2000). Subcortical and cortical brain activity during the feeling of self-generated emotions. Nature Neuroscience, 3, 1049-1056.

D’Esposito, M., Zarahn, E., \& Aguirre, G. K. (1999). Event-related functional MRI: Implications for cognitive psychology. Psychological Bulletin, 125, 155-164.

DeLongis, A., Folkman, S., \& Lazarus, R. S. (1988). The impact of daily stress on health and mood: Psychological and social resources as mediators. Journal of Personality and Social Psychology, 54, 486-495.

Elliott, R., Sahakian, B. J., Michael, A., Paykel, E. S., \& Dolan, R. J. (1998). Abnormal neural response to feedback on planning and guessing tasks in patients with unipolar depression. Psychological Medicine, 28, 559-571.

Fox, P. T., \& Raichle, M. E. (1986). Focal physiological uncoupling of cerebral blood flow and oxidative metabolism during somatosensory stimulation in human subjects. Proceedings of the National Academy of Sciences of the United States of America, 83, 1140-1144.

Fu, C. H., Williams, S. C., Cleare, A. J., Brammer, M. J., Walsh, N. D., Kim, J. et al. (2004). Attenuation of the neural response to sad faces in major depression by antidepressant treatment: A prospective, event-related functional magnetic resonance imaging study. Archives of General Psychiatry, 61, 877-889.

George, M. S., Ketter, T. A., \& Post, R. M. (1994). Activation studies in mood disorders. Psychiatric Annals, 24, 648-652.

George, M. S., Ketter, T. A., Parekh, P. I., Horwitz, B., Herscovitch, P., \& Post, R. M. (1995). Brain activity during transient sadness and happiness in healthy women. American Journal of Psychiatry, 152, 341-351.

Harmer, C. J., Mackay, C. E., Reid, C. B., Cowen, P. J., \& Goodwin, G. M. (2006). Antidepressant drug treatment modifies the neural processing of nonconscious threat cues. Biological Psychiatry, 59, 816-820. 
Correlatos Anatômico-Funcionais das Emoções Mapeados com Técnicas de...

Lane, R. D., Reiman, E. M., Ahern, G. L., Schwartz, G. E., \& Davidson, R. J. (1997). Neuroanatomical correlates of happiness, sadness, and disgust. American Journal of Psychiatry, 154, 926-933.

Lang, P. J., Bradley, M. M., \& Cuthbert, B. N. (2001). International affective picture system (IAPS): Instruction manual and affective ratings. Technical Report A-5. The Center for Research in Psychophysiology. University of Florida.

Markowitsch, H. J., Vandekerckhove, M. M., Lanfermann, H., \& Russ, M. O. (2003). Engagement of lateral and medial prefrontal areas in the ecphory of sad and happy autobiographical memories. Cortex, 39, 643-665.

Marks, D. F. (1989). Construct validity of the Vividness of Visual Imagery Questionnaire. Perception and Motor Skills, 69(2), 459-465.

Mayberg, H. S., Liotti, M., Brannan, S. K., Mcginnis, S., Mahurin, R. K., Jerabek, P. A. et al. (1999). Reciprocal limbic-cortical function and negative mood: Converging PET findings in depression and normal sadness. American Journal of Psychiatry, 156, 675682.

Mayberg, H. S., Brannan, S. K., Tekell, J. L., Silva, J. A., Mahurin, R. K., McGinnis, S. et al. (2000). Regional metabolic effects of fluoxetine in major depression: Serial changes and relationship to clinical response. Biological Psychiatry, 48, 830-843.

Moll, J., de Oliveira-Souza, R., Moll, F. T., Bramati, I. E., \& Andreiuolo, P. A. (2002). The cerebral correlates of set-shifting: An fMRI study of the trail making test. Arquivos de Neuropsiquiatria, 60, 900-905.

Montaldi, D., Brooks, D. N., McColl, J. H., Wyper, D. J., Patterson, J., Barron, E. et al. (1990). Measurements of regional cerebral blood flow and cognitive performance in Alzheimer's disease. Journal of Neurology, Neurosurgery and Psychiatry, 53, 33-38.

Morris, J. S., Frith, C. D., Perrett, D. I., Rowland, D., Young, A. W., Calder, A. J. et al. (1996). A differential neural response in the human amygdala to fearful and happy facial expressions. Nature, 383, 812-815.

Nolde, S. F., Johnson, M. K., \& D’Esposito, M. (1998). Left prefrontal activation during episodic remembering: An event-related fMRI study. Neuroreport, 9, 3509-3514.

Ogawa, S., Lee, T. M., Kay, A. R., \& Tank, D. W. (1990). Brain magnetic resonance imaging with contrast dependent on blood oxygenation. Proceedings of the National Academy of Sciences of the United States of America, 87, 9868-9872.

Pardo, J. V., Pardo, P. J., \& Raichle, M. E. (1993). Neural correlates of self-induced dysphoria. American Journal of Psychiatry, 150, 713-719. 
Phan, K. L., Wager, T., Taylor, S. F., \& Liberzon I. (2002). Functional neuroanatomy of emotion: A meta-analysis of emotion activation studies in PET and fMRI. Neuroimage, 16, 331-348.

Phillips, M. L., Young, A. W., Senior, C., Brammer, M., Andrew, C., Calder, A. J. et al. (1997). A specific neural substrate for perceiving facial expressions of disgust. Nature, 389, 495-498.

Phillips, M. L., Marks, I. M., Senior, C., Lythgoe, D., O’Dwyer, A. M., Meehan, O. et al. (2000). A differential neural response in obsessive-compulsive disorder patients with washing compared with checking symptoms to disgust. Psychological Medicine, 30, 1037-1050.

Phillips, M. L., Drevets, W. C., Rauch, S. L., \& Lane, R. (2003a). Neurobiology of emotion perception I: The neural basis of normal emotion perception. Biological Psychiatry, 54, 504-514.

Phillips, M. L., Drevets, W. C., Rauch, S. L., \& Lane, R. (2003b). Neurobiology of emotion perception II: Implications for major psychiatric disorders. Biological Psychiatry, 54, 515-528.

Raichle, M. E. (1987). Circulatory and metabolic correlates of brain function in normal humans. In F. Plum (Ed.), The nervous system: Higher functions of the brain (pp. 643674). Bethesda, MD: American Physiological Society.

Rauch, S. L., Savage, C. R., Alpert, N. M., Fischman, A. J., \& Jenike, M. A. (1997). The functional neuroanatomy of anxiety: A study of three disorders using positron emission tomography and symptom provocation. Biological Psychiatry, 42, 446-452.

Roy, C. S., \& Sherrington, C. S. (1890). On the regulation of the blood supply of the brain. Journal of Physiology, 11, 85-108.

Saxena, S., Brody, A. L., Schwartz, J. M., \& Baxter, L. R. (1998). Neuroimaging and frontalsubcortical circuitry in obsessive-compulsive disorder. British Journal of Psychiatry, $173,26-37$.

Schaefer, H. S., Putnam, K. M., Benca, R. M., \& Davidson, R. J. (2006). Event-related functional magnetic resonance imaging measures of neural activity to positive social stimuli in pre- and post-treatment depression. Biological Psychiatry, 60, 974-986.

Senaha, M. L. H., Martin, M. G. M., Amaro Jr., E., Campi, C., \& Caramelli, P. (2005). Patterns of cerebral activation during lexical and phonological reading in Portuguese. Brazilian Journal of Medical and Biological Research, 38, 1847-1856.

Stein, M. B., Goldin, P. R., Sareen, J., Zorrilla, L. T., \& Brown, G. G. (2002). Increased amygdala activation to angry and contemptuous faces in generalized social phobia. Archives of General Psychiatry, 59, 1027-1034. 
Vuilleumier, P., \& Pourtois, G. (2007). Distributed and interactive brain mechanisms during emotion face perception: Evidence from functional neuroimaging. Neuropsychologia, 45, 174-194.

Whalen, P. J., Rauch, S. L., Etcoff, N. L., McInerney, S. C., Lee, M. B., \& Jenike, M. A. (1998). Masked presentations of emotional facial expressions modulate amygdala activity without explicit knowledge. Journal of Neuroscience, 18, 411-418.

Weinberger, D. R., Berman, K. F., \& Zec, R. F. (1986). Physiologic dysfunction of dorsolateral prefrontal cortex in schizophrenia I. Regional cerebral blood flow evidence. Archives of General Psychiatry, 43, 114-124.

Wheeler, M. E., Shulman, G. L., Buckner, R. L, Miezin, F. M., Velanova, K., \& Petersen, S. E. (2006). Evidence for separate perceptual reactivation and search processes during remembering. Cerebral Cortex, 16, 949-959.

Woods, S. W., Hegeman, I . M., Zubal, I. G., Krystal, J. H., Koster, K., Krystal, J. H. et al. (1991). Visual stimulation increases technetium-99m-HMPAO distribution in human visual cortex. Journal of nuclear medicine, 32, 210-215.

Recebido em: 17/12/2006

Aceito em: 20/12/2006 